\title{
Dual Implication of 2',3'-Cyclic Nucleotide 3' Phosphodiesterase as Major Autoantigen and C3 Complement-binding Protein in the Pathogenesis of Multiple Sclerosis
}

\author{
Michael J. Walsh and Jacinta M. Murray \\ Department of Neurology, Mount Sinai School of Medicine, New York 10029
}

\begin{abstract}
Multiple sclerosis (MS) is characterized by intra-blood-brain barrier immunoglobulin synthesis that persists lifelong. Subcellular fractionation and two-dimensional electrophoresis were used in conjunction with immune precipitation and immunoblotting to identify antigenic determinants for this immunoglobulin. We report that $2^{\prime}, 3^{\prime}$-cyclic nucleotide $3^{\prime}$ phosphodiesterase (CNP), a protein associated with oligodendrocyte/myelin membranes, also present in lymphocytes and retina, is one major target for the humoral response. Antibodies to CNP are detected in sera of $74 \%$ of MS patients. The antibodies are IgM and are present in serum in high titer as well as in cerebrospinal fluid. The antibody response is temporally persistent, consistent with systemic immune activation and persistent antigenic stimulation. Moreover, CNP is isolated as an immune complex from MS brain. CNP is expressed as two isoforms, with CNPII identical to CNPI but with a 20-amino acid extension at the amino terminus of CNPII; however, the antibody response is exclusively restricted to CNPI. In contrast, both isoforms bind the $\mathrm{C} 3$ complement, providing a plausible mechanism in MS central nervous system (CNS) for opsonization of myelin membrane $\mathrm{CNP}$, mediated via the $\mathrm{C} 3$ receptor, and phagocytosis of CNP-Ig immune complexes, mediated by membrane Ig Fc receptors of macrophages and CNS microglia. (J. Clin. Invest. 1998. 101:1923-1931.) Key words: multiple sclerosis - microglia $・ 2^{\prime}, 3^{\prime}$-cyclic nucleotide $3^{\prime}$-phosphodiesterase $\bullet$ complement $\bullet$ IgM antibodies

\section{Introduction}

A notable aspect of multiple sclerosis (MS) ${ }^{1}$ is robust and temporally persistent intra-blood-brain barrier (BBB) Ig synthe-

Address correspondence to Michael J. Walsh, M.D., Box 1065, Neurology Department, Mount Sinai Hospital, One Gustave Levy Place, New York, NY 10029. Phone: 212-241-5320; FAX: 212-427-7420; E-mail: mwalsh@msvax.mssm.edu

Received for publication 10 October 1997 and accepted in revised form 23 February 1998.

1. Abbreviations used in this paper: $\mathrm{BBB}$, blood-brain barrier; CNP, $2^{\prime}, 3^{\prime}$-cyclic nucleotide $3^{\prime}$-phosphodiesterase; CSF, cerebrospinal fluid; 2DE, two-dimensional electrophoresis; MBP, myelin basic protein; MS, multiple sclerosis; NEpHGE, nonequilibrium $\mathrm{pH}$ gradient gel electrophoresis; OL, oligodendrocyte; OND, other neurological diseases; PNS, peripheral nervous system; WF, Wolfgram fraction.

J. Clin. Invest.

(C) The American Society for Clinical Investigation, Inc. 0021-9738/98/05/1923/09 \$2.00

Volume 101, Number 9, May 1998, 1923-1931

http://www.jci.org sis. The IgG is mainly IgG1 subclass and shows light chain, allotype, and idiotype restriction. By isoelectric focusing, the cerebrospinal fluid (CSF) IgG of MS patients resolves into discrete fractions designated oligoclonal IgG bands. Compared with autologous serum IgG, the CSF bands are more cathodic in mobility. These two features, oligoclonal banding and cathodic shift, are highly characteristic, occur in $>90 \%$ of all MS patients, and are strongly supportive of the diagnosis of MS in an appropriate clinical setting $(1,2)$. Although predominantly $\mathrm{IgG}$, intra-BBB synthesis of $\operatorname{IgA}$ and $\operatorname{IgM}$ has also been observed; the response is oligoclonal for each major Ig class, is temporally persistent, and evinces remarkable clonal invariance, consistent with ongoing and specific antigenic stimulation $(3,4)$. Antibodies to several antigens have been reported in MS (see reference 5); however, such antibodies are found in low titer, their specificity and sensitivity are low, and none have clinical value as indicative of disease severity or prognosis. Thus, MS is distinguished from the most common putative autoimmune disorders by the absence of a specific antibody marker that has clinical utility. Consequently, at present, the evaluation of disease severity and progression or monitoring of agents as MS treatments is reliant not on the measurement of any immunological parameter but on high-technology investigations, particularly magnetic resonance imaging to assess disease burden and drug effects. In fact, the favorable results of magnetic resonance imaging analyses were important for the expedited approval of the various interferons as MS treatments (6).

Identification of major antigen targets for intra-BBB-synthesized Ig has been unproductive but remains an important objective of MS research. First, in many infectious central nervous system (CNS) disorders, much of the CSF Ig is directed at the causative organism; thus, in MS, intra-BBB-synthesized Ig is a putative probe to attempt isolation of an infectious agent. Second, the intra-BBB Ig synthesis that is a feature of many infectious and inflammatory diseases of the CNS tends to abate or disappear with effective treatment; thus, the modulation of intra-BBB Ig synthesis in MS might be one type of monitor for response to experimental therapies. Third, identification of antigen targets against which the Ig is directed would provide scientific direction for the $\mathrm{T}$ cell determinants that might be pursued most profitably in MS. This is based on the experience with numerous other putative autoimmune disorders where antibody studies have provided the conceptual basis for $\mathrm{T}$ cell studies as well as therapeutic interventions, most remarkably and productively recently for insulin-dependant diabetes mellitus $(7,8)$. This formulation may be equally applicable to reports for involvement of specific viruses in MS. Major roles for infectious agents detected recently in MS as well as normal CNS, i.e., human herpes virus 6 (9) or herpes simplex 1 and 2 (10), would be more plausible if vigorous intra-BBB humoral immune responses especially of the IgM class were demonstrable and persistent to these agents uniquely in MS patients.

We have begun to approach fundamental aspects of the im- 
mune response in MS using molecular immunological approaches combined with Edman chemical sequencing and mass spectrometry to characterize peptides and other molecules complexed with Ig, MHC molecules, and heat shock proteins, the three multigene families that may be involved in specific antigen presentation in MS CNS (1). Using these approaches, we have found some strong reactions of MS CSF and sera with several brain proteins. Here, we report that $2^{\prime}, 3^{\prime}$ cyclic nucleotide $3^{\prime}$-phosphodiesterase (EC 3.1.4.37, CNP) is one major target for the humoral response in MS. CNP is a membrane-associated protein of the oligodendrocyte (OL) $(11,12)$, but is also expressed at high levels by lymphocytes (13) and in retina (14). CNPI (46 kD) and CNPII (48 kD) are derived by alternative splicing from a single gene, and are identical except for an additional 20 amino acids at the amino terminus of CNPII (15). Both CNPII and CNPI are highly modified posttranslationally and differentially, including serine/ threonine phosphorylation, palmitoylation, and isoprenylation, and are heterogenous in size and charge $(11,12,16,17)$. The antibody response is directed against CNPI, whereas both isoforms, but particularly CNPII, bind avidly to C3, a pivotal protein of the complement system highly implicated in MS (18) and its experimental models (19). CNP is also isolated as an immune complex from MS CNS, indicating that this humoral response may have relevance in vivo. Finally and unexpectedly, the response to CNP is chiefly $\operatorname{IgM}$, is temporally persistent, and is present in serum as well as CSF. This indicates that as for the cellular immune response $(20,21)$, pronounced humoral immune activation in MS also occurs systemically even if the major clinical and pathological consequences are manifest exclusively in the CNS.

\section{Methods}

Patients and brain and spinal cord specimens. CSF and serum were obtained from patients with clinically definite MS and patients with other neurological diseases (OND). Normal blood donor sera were obtained from the Blood Bank at Mount Sinai School of Medicine. Sera from patients with SLE were generously provided by V. Hanson (Mount Sinai School of Medicine) and F. Medina (Centro Médico Nacional Siglo XXI, Mexico City, Mexico). Brain tissue from patients without neurological disease and with MS, as well as human sciatic nerve for the isolation of peripheral nervous system (PNS) myelin, was obtained from the National Neurological Research Specimen Bank (Los Angeles, CA).

Antibodies and reagents. Rabbit polyclonal antisera to myelin proteins and $\mathrm{mAb}$ to myelin/OL glycoprotein were obtained from $\mathrm{D}$. Colman and A. Gow (Mount Sinai School of Medicine); rabbit polyclonal to PMP22 was obtained from G. Jackson Snipes (Stanford University, Stanford, CA); $\mathrm{mAb}$ to CNP and myelin basic protein (MBP) were obtained from Sigma Chemical Co. (St. Louis, MO). mAb and polyclonal antibodies to complement proteins were obtained from Sigma Chemical Co., Quidel (San Diego, CA), and DAKO Corp. (Carpinteria, CA). Alkaline phosphatase-coupled anti-human IgG, $\mathrm{IgA}$, and IgM were obtained from Sigma Chemical Co.

Gel electrophoresis and immunoblotting. The procedures for SDSPAGE, two-dimensional electrophoresis (2DE), nonequilibrium $\mathrm{pH}$ gradient gel electrophoresis (NEpHGE) (22), and immunoblotting have been described $(3,23)$. CNS and PNS myelin were isolated by sucrose gradient centrifugation (24) except that all buffers contained $1 \mathrm{mM}$ DTT and PMSF, 1,10-phenanthroline, EDTA, and EGTA, each at $1 \mathrm{mM}$, and pepstatin and leupeptin, each at $20 \mathrm{mg}$ and $15 \mathrm{mg} /$ liter (25). CNS myelin was further fractionated by homogenization in chloroform/methanol (13) followed by centrifugation to obtain a
Wolfgram fraction (WF) highly enriched in CNPII/I, which was dried and suspended in lysis buffer containing $10.2 \mathrm{M}$ urea with $2 \% \mathrm{NP}-40$ and $1 \%$ DTT. Constituent proteins were resolved by NEpHGE with omission of equilibration of the first dimension tube gel in SDS sample buffer (23). Procedures for silver staining and immunoblotting were as described $(3,23)$.

Isolation of Ig from MS brain. A protocol designed for affinity isolation of MHC class II molecules was used for brain fractionation (26). Frozen tissue was broken in 4-6-g pieces, chopped into 1-2-mm fragments, transferred immediately into ice-cold buffer, and homogenized with 10 complete up and down strokes using a motor-driven Teflon glass homogenizer set at $850 \mathrm{rpm}$. The homogenization buffer was $10 \mathrm{mM}$ Tris $\mathrm{HCl}, \mathrm{pH} 8$, with $1 \mathrm{mM}$ DTT, and PMSF, 1,10-phenanthroline, EDTA, EGTA, each at $1 \mathrm{mM}$, and pepstatin and leupeptin, each at $20 \mathrm{mg}$ and $15 \mathrm{mg} /$ liter (buffer I). The homogenate was centrifuged at 2,000 $\mathrm{g}$ for $10 \mathrm{~min}$. The supernatants were decanted and saved, and the pellet was resuspended in buffer I and spun down again; this procedure was repeated six times. The combined supernatants were centrifuged at $120,000 \mathrm{~g}$ for $60 \mathrm{~min}$. The pellets were combined and suspended in buffer I by homogenization, adjusted to $4 \%$ NP-40, and stirred on ice for $30 \mathrm{~min}$ to extract the membrane proteins. The suspension was centrifuged at $120,000 \mathrm{~g}$ for $120 \mathrm{~min}$ to obtain the NP-40 extract, which was then passaged over a protein A-Sepharose column to recover the bulk of the solubilized IgG, and an anti-IgGAM immunoabsorbent to recover IgA and IgM as well as $\mathrm{IgG}$ that was not bound by protein A. The column wash conditions were as described (26). Protein bound to the affinity resins were eluted with $0.1 \mathrm{~N}$ acetic acid, $\mathrm{pH} 2.9$, frozen immediately, and lyophilized.

Characterization of $C 3$ interactions with CNP. The WF, resolved by NEpHGE, was blotted to nitrocellulose; CNPI and CNPII were excised separately using 8-10 NEpHGE blots for each experiment, blocked with $0.5 \%$ polyvinyl-pyrrolidone in $0.1 \mathrm{~N}$ acetic acid (27), washed 10 times with water, and incubated with serum from healthy donors or from MS patients (100 $\mu \mathrm{l}$ serum diluted to $1.2 \mathrm{ml}$ with 50 $\mathrm{mM}$ Tris $\mathrm{HCl}, \mathrm{pH} 8$, containing $150 \mathrm{mM} \mathrm{NaCl}$, and $0.1 \%$ sodium azide at $25^{\circ} \mathrm{C}$ for $4 \mathrm{~h}$ or overnight at $4^{\circ} \mathrm{C}$ ). The nitrocellulose pieces were washed 10 times with $50 \mathrm{mM}$ Tris $\mathrm{HCl}, \mathrm{pH} 8$, with $250 \mathrm{mM}$ $\mathrm{NaCl}, 0.2 \%$ Tween 20 , five times with water, and bound proteins were recovered with the following eluants: (a) $500 \mu \mathrm{l} 2 \mathrm{~N}$ acetic acid with heating at $100^{\circ} \mathrm{C}$ for $5 \mathrm{~min} ;(b) 10 \mathrm{mM}$ Tris $\mathrm{HCl}, \mathrm{pH}$, with $0.5 \%$ SDS, $10 \mathrm{mM}$ DTT, and $1 \%$ glycerol at $100^{\circ} \mathrm{C}$ for $4 \mathrm{~min}$; and (c) $1 \mathrm{M}$ hydroxylamine, $\mathrm{pH} 11.5$, with or without $0.5 \%$ SDS. The eluates were lyophilized or precipitated with methanol and subjected to SDSPAGE followed by silver staining or immunoblotting to identify specific bound proteins.

\section{Results}

NEpHGE of the WF and immunoblot analysis. The WF was extracted from myelin isolated from normal and MS brain, then separated by SDS-PAGE, 2DE, and NEpHGE, resolving several hundred proteins including MS candidate autoantigens MBP, proteolipid protein, myelin-associated glycoprotein, myelin/OL glycoprotein, the heat shock proteins, and transaldolase (see reference 5). NEpHGE was found optimal for resolution of the proteins of the myelin fraction, many of which are highly positively charged (Fig. 1). Fig. 2 shows the characterization of $\mathrm{CNP}$ in the WF from CNS myelin, demonstrating that the CNPI/II isoforms are present in human CNS myelin at $\approx 1: 1$ stoichiometry as shown by protein staining (Fig. $2 a$ ) and by immunoblotting with an antibody to CNP (Fig. $2 b$ ). This NEpHGE-resolved myelin or WF was blotted and probed with CSF and sera from MS and OND patients or sera from normal donors. Fig. 3, $a-c$, is representative, illustrating results 


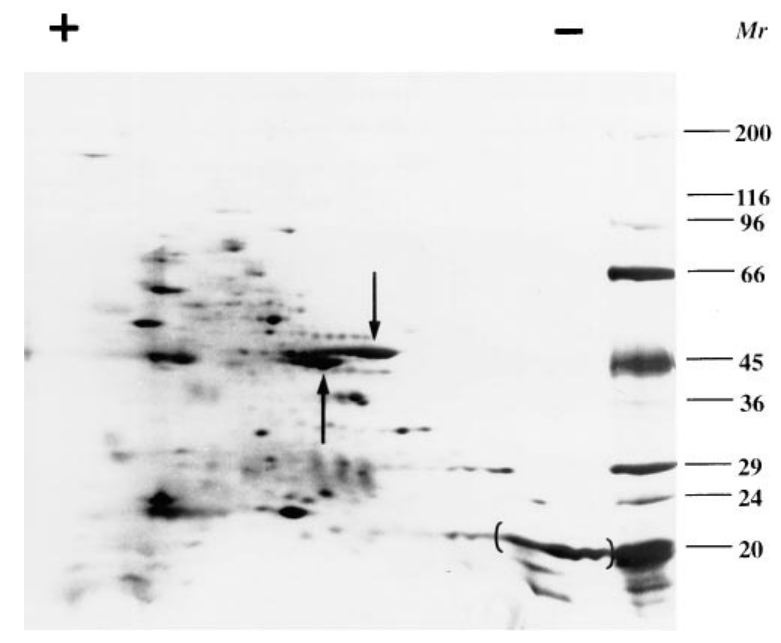

Figure 1. NEpHGE separation of proteins of the WF of human CNS myelin (60 $\mu \mathrm{g}$ protein). Arrows, CNPI ( $\uparrow)$ and CNPII $(\downarrow)$, respectively. Brackets, Position of MBP. Bars, Molecular masses of marker proteins from 200 to $20 \mathrm{kD}$. In this and the other figures, the cathode $(-)$ is on the right.

for one MS patient using CSF (diluted 1:10) and serum (diluted 1:2,000 or 1:5,000) and showing an intense reaction directed almost exclusively against the CNPI isoform. This result was surprising, because the entire primary sequence of CNPI is included within CNPII, and it suggests that the epitope recognized is a posttranslational modification unique to the blocked amino terminus of CNPI (13), or lipid modifications including palmitoylation and isoprenylation unique to one CNP isoform $(16,17)$, or other modifications still unidentified.

Affinity chromatographic isolation of CNP-Ig immune complexes from $M S$ brain. We wanted to see if Ig-CNP immune complexes could be isolated from MS CNS as a confirmation of the immunoblot results, and under conditions where there is not significant protein denaturation, as occurs with the NEpHGE procedure or SDS-PAGE. The MS CNS membrane fraction solubilized in buffer I with $4 \%$ NP-40 was passaged sequentially over protein $\mathrm{A}$ and anti-human polyvalent $\operatorname{Ig}(\mathrm{G}, \mathrm{A}$, and M) affinity columns. MS CNS HLA-DR was also isolated using an $\mathrm{mAb}$ resin as part of the procedure (26). After rigorous column washes with stringency adequate for purification

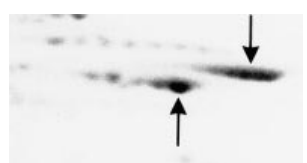

a

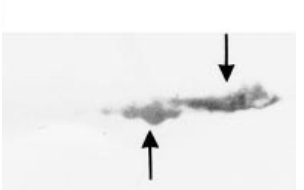

b

Figure 2. NEpHGE separation and immunoblot analysis of proteins of the WF of human CNS myelin. Portions of gels stained for protein indicate the positions of CNPI $(\uparrow)$ and CNPII $(\downarrow)$, respectively (a). CNP was detected using rabbit polyclonal antibody to CNP $(b)$. of the HLA-DR heterodimer to homogeneity, with evidence for physiologically bound peptides as indicated by SDS-resistant heterodimers (28) (our unpublished results), proteins bound to each affinity resin were eluted. Eluates from the antiIg immunoabsorbent or protein A were resolved by SDSPAGE, blotted, and probed with an mAb to CNP. Even though most of CNS CNP is not solubilized under these conditions by virtue of its tight association with actin-based cytoskeletal elements (29), CNP is recovered in the eluate from the anti-Ig immunoabsorbent (Fig. $3 d$ ). In contrast, parallel blots probed with antibody to MBP were negative.

Antibody to CNPI is predominantly IgM and is detected in serum and CSF. Our preliminary studies on antibody reactivities to the myelin fraction in MS which resulted in the detection of the anti-CNPI response had used CSF on the premise that intra-BBB-synthesized IgG is most highly enriched in putative specific antibodies (4). However, comparative immunoblot analysis using MS CSF and autologous serum showed that the response was also readily detected in serum. Immunodetection with antibodies specific for $\mathrm{IgG}, \mathrm{A}$, or M showed that the predominant MS response to CNPI was IgM, with a contribution by $\operatorname{IgA}$ and no reaction for $\operatorname{IgG}$ (Fig. 4, $a-c$ ). This result is also indicative of a response to a nonpeptide modification of CNPI. The IgM concentration in CSF is $10^{-3}-10^{-5}$ that of serum even in the presence of intra-BBB IgM synthesis as occurs in MS $(3,30)$. Thus, larger volumes of CSF than are usually available would be required using NEpHGE blots. Therefore, sera were used for the subsequent analyses reported here.

$A b$ response to CNPI in MS and other diseases. The results described here were obtained using sera diluted to 1:250. NEpHGE blots of the WF were probed with sera from 70 pa-
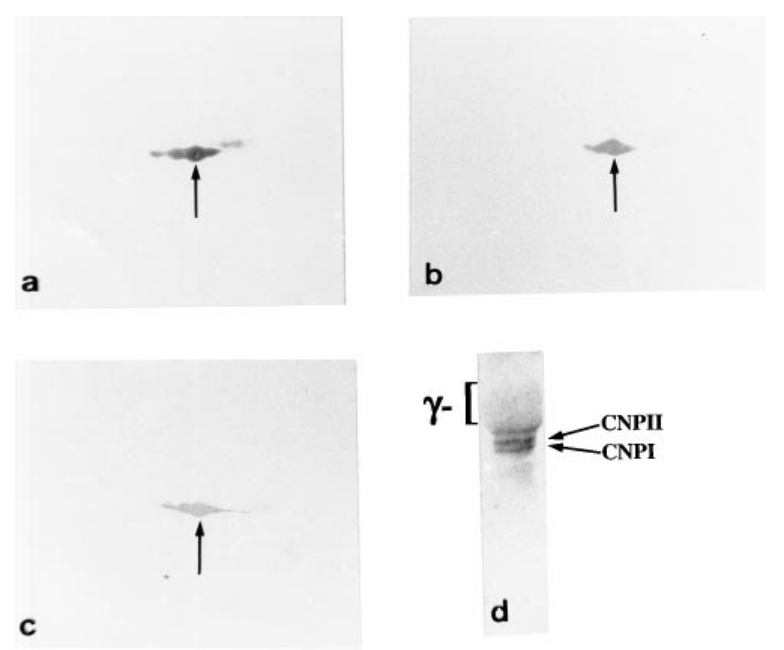

Figure 3. Immunoblot analysis showing reaction of MS CSF and serum with CNP $(a-c)$ and coisolation of CNP with Ig solubilized from MS CNS membrane extracts $(d)$. NEpHGE-resolved WF proteins (30 $\mu \mathrm{g}$ protein) were blotted and incubated overnight with MS CSF diluted to 1:10 $(a)$, or autologous serum diluted to 1:2,000 $(b)$ or 1:5,000 (c). Alkaline phosphatase-coupled goat anti-human IgM (Sigma Chemical Co.) $(1: 10,000)$ was used to detect the immune reaction. The arrows in $a-c$ point to CNPI. (d) Ig isolated from the membrane fraction of MS CNS was resolved by SDS-PAGE and blotted, and copurifying CNP was detected with specific $\mathrm{mAb}$. The position of the $\operatorname{IgG~H}$ chain $(\gamma)$ is indicated; the two arrows point towards CNPI/II. 

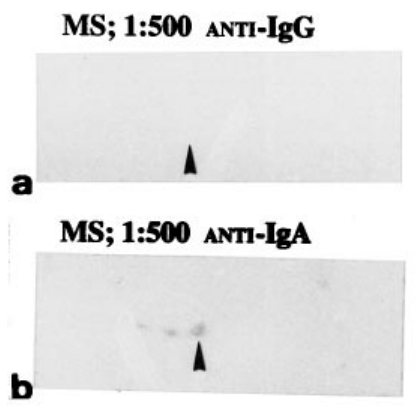

MS; 1:2,500 ANTI-IgM

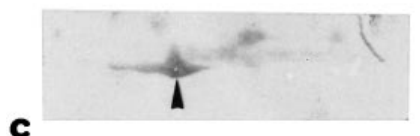

NORMAL; 1:1,000 ANTI-IgM
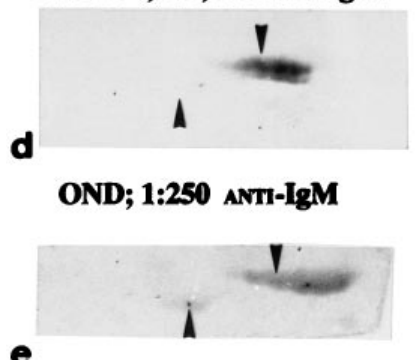
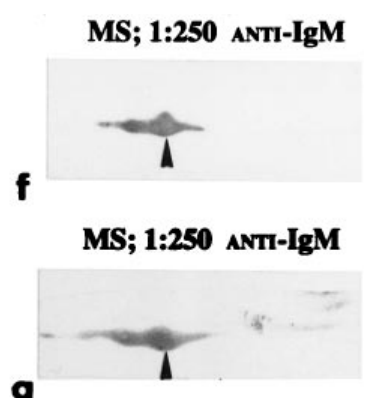

$\mathbf{g}$

MS; 1:250 ANTI-IgM

h

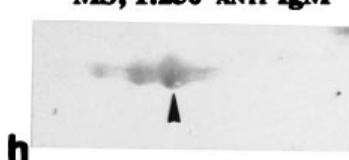

MS; 1:1,000 ANT-IgM

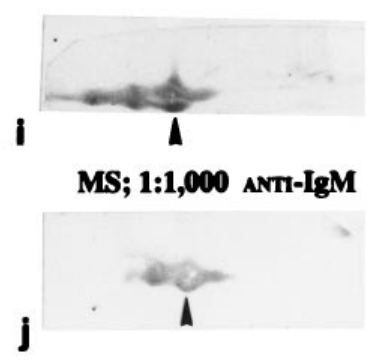

Figure 4. Immunoblot analysis of NEpHGE-resolved WF $(\approx 30 \mu \mathrm{g}$ protein) showing reaction of MS serum with CNP detected using alkaline phosphatase-coupled goat anti-human $\operatorname{IgG}(a)$, anti-human $\operatorname{IgA}(b)$, or anti-human $\operatorname{IgM}(c) ; d$ is a normal blood donor, and $e$ is a patient with viral meningitis $(O N D) ; f-j$ are sera from five MS patients. Up arrowheads point to CNPI; down arrowheads point to CNPII. The reaction of MS sera with CNPI occurs within minutes, allowing blot development to be terminated promptly $(f-j)$; in $d$ and $e$, blot development was prolonged for several hours during which time the intense staining of CNPII became apparent. In $c-j$, the alkaline phosphatase-coupled goat anti-human IgM (Sigma Chemical Co.) was used at 1:10,000.

tients with MS, and 52 (74\%) patients from this total gave an unequivocal and strong reaction with CNP, exclusively or predominantly CNPI (Fig. 4, $f-j$ ). To establish the disease specificity of this result for MS, we examined the response to CNP using sera from 61 individuals including 33 OND patients, 16 patients with SLE (10 of whom had prominent neurological or neuropsychiatric manifestations), and 28 healthy blood donors. The OND population included patients with various CNS infections (e.g., coccidioidomycosis, subacute sclerosing panencephalitis, herpes zoster encephalitis, neurosyphilis, neurotuberculosis, and viral meningitis of undetermined etiology) as well as patients with neurodegenerative and cerebrovascular disorders (Table I). Representative NEpHGE blot results for control (Fig. $4 d$ ) and OND sera (Fig. $4 e$ ) are shown. None of the 28 healthy donors ever gave a response that mimicked MS, i.e., no individual gave the pattern illustrated in Fig. 3, $a-c$, or Fig. 4, $f-j$, showing a predominant or exclusive reaction with CNPI. Two OND sera gave a prompt reaction with CNPI with sera diluted to 1:250, but an equal response to both isoforms when sera were tested diluted to 1:5,000; the diagnoses were

Charcot-Marie-Tooth disease and the primary lateral sclerosis form of motor neuron disease. One multiple-transfused OND patient with recurring attacks of bacterial endocarditis and neurologic episodes that included visual loss considered to be due to thromboembolism gave a reaction with CNPI similar to MS. The current protocol for definitive detection of anti-CNPI antibody (i.e., subcellular fractionation, NEpHGE separation of the proteins of the WF, and immunoblotting) is cumbersome for examination of each patient's response to CNPI using serial dilution of sera since each test requires a new blot. Therefore, we selected the MS sera that gave the most rapid and robust responses to testing using sera diluted to 1:250 for examination of their responses at higher dilutions as illustrated in Figs. 3 and 4. Within this selected MS population, 17 patients were positive at 1:5,000, 11 of 17 were also positive on retesting at 1:10,000, and 10 of 11 were still positive at 1:20,000. Sera of eight MS patients were also examined at multiple time points extending over $10 \mathrm{yr}$ to determine if there were temporal fluctuations in IgM immunoreactivity. Sequential immunoblot analyses showed that the anti-CNPI response was persistent in all cases.

Epitope density and the immune response to CNPI. Before use of NEpHGE for the studies reported earlier, we had begun screening for immunoreactivity using SDS-PAGE-resolved normal or MS CNS fractions including whole myelin and the WF. A robust and easily interpretable reaction with CNPI was not observed when protein fractions resolved by SDS-PAGE were blotted and then probed with MS CSF or sera. To examine if this was partly due to the harsher conditions of protein denaturation used for SDS-PAGE (i.e., 4\% SDS), NEpHGEresolved and nitrocellulose-immobilized CNP (only exposed to $0.1 \%$ SDS in the second dimension) was preincubated with SDS sample buffer at $25^{\circ} \mathrm{C}$ for $30 \mathrm{~min}$. The blot was washed to remove residual SDS and then probed with MS sera. A strong

Table I. Disease Diagnoses for the Non-MS Patients

\begin{tabular}{lc}
\hline \multicolumn{1}{c}{ Diagnosis } & No. of patients \\
\hline CNS coccidioidomycosis & 1 \\
Mycoplasma-associated myelopathy & 1 \\
HIV & 1 \\
Neurosyphilis & 1 \\
Subacute sclerosing panencephalitis & 2 \\
Neurotuberculosis & 1 \\
Guillain-Barre syndrome & 1 \\
Bacterial endocarditis & 1 \\
Herpes zoster encephalitis & 1 \\
Viral meningitis & 1 \\
SLE & 16 \\
Polyneuropathy/monoclonal gammopathy & 1 \\
Alzheimer's disease & 5 \\
Cerebellar ataxia & 3 \\
Familial polyneuropathy & 1 \\
Wilson's disease & 1 \\
Motor neuron disease & 1 \\
CNS neoplasm & 3 \\
Cerebrovascular disease & 4 \\
Headache & 1 \\
Peripheral neuropathies & 2 \\
\hline
\end{tabular}


reaction of MS sera with CNPI was still demonstrable (data not shown). The comparative clarity of the immune reaction of MS CSF and serum with NEpHGE as illustrated earlier (Fig. $3, a-c)$ is most likely due to the clear resolution of CNP away from the numerous comigrating proteins in this size range and the increased "epitope density" possible with the greater loading capacity of $2 \mathrm{DE} / \mathrm{NEpHGE}$. There is precedence for the importance of epitope density especially with $\operatorname{IgM}$ antibodies. The affinity of rheumatoid factor was several orders of magnitude greater for aggregated human IgG compared with IgG monomer (31). The importance of aggregation state and epitope density has been observed repeatedly especially in regard to detection of immune reactions important in neurological practice, e.g., for detection of antiphospholipid (32) antiglycolipid antibodies (33), and in autoimmune neuropathies associated with antiganglioside antibodies (see reference 34) where typically $200 \mathrm{pmol}-1 \mathrm{nmol}$ of individual ganglioside is used for each thin layer chromatographic immunoassay.

Interaction of human sera and secondary antibody reagents with CNPII. In the systematic analysis of the response to CNP as reported above, two observations were made which indicated that there might be further mechanism(s) of interaction of sera with CNP completely separable from the immunologically specific reaction of MS sera with CNPI. First, we noted that incubation of NEpHGE blots with sera from the three sources used in this study (i.e., normal blood donors, and OND and MS patients) at lower dilutions (e.g., 1:250-1:1,000) stained CNPII especially if the blot development was prolonged; this phenomenon is illustrated in Fig. 4, $d$ and $e$. Because of the preferential staining of CNPII but not CNPI despite the fact that about equal amounts of the CNP isoforms were immobilized on the blot, we did not dismiss the reaction as entirely nonspecific. Second, in a series of control experiments to examine each step of our protocol for detection of anti-CNPI antibody, secondary antibody reagents used to detect immune complexes were incubated with NEpHGE blots but without prior incubation with human sera. As illustrated in Fig. $5 a$, staining of CNPII is still apparent when secondary antibody was used at a dilution of 1:750. This staining with enzyme-coupled antisera was not observed when we modified the protocol to detect immune reactions with CNPI and diluted the antisera to 1:5,000-1:10,000. After development, the blot shown in Fig. $5 a$ was photographed and then reincubated with $\mathrm{mAb}$ to CNP to demonstrate unequivocally that the failure of the secondary antibody to show a prominent reaction with CNPI was not due to a lesser amount of CNPI on this blot compared with CNPII (Fig. $5 \mathrm{~b}$ ). The converse situation was also examined, i.e., the blot was first incubated with MS serum known to give a robust reaction with CNPI, the immune complex was detected with alkaline phosphatase-coupled IgM (diluted 1:10,000, thus obviating reaction with CNPII; shown in Fig. $5 a$ where the antiserum was used at 1:750), and the blot was photographed (Fig. $5 c$ ) and then reincubated with anti-CNP mAb to show that both CNP isoforms were present on the blot (Fig. $5 d$ ).

CNP binds the C3 component of complement. The binding of normal sera or secondary antibodies to CNP, predominantly CNPII rather than CNPI (Fig. $4, d$ and $e$, and Fig. $5 a$, respectively), even if the converse of the pattern observed in MS, was a potential complicating factor to detection of the anti-CNPI response, e.g., the development of an ELISA for routine clinical application. We and other investigators had previously identified complement proteins, most notably C3 and various

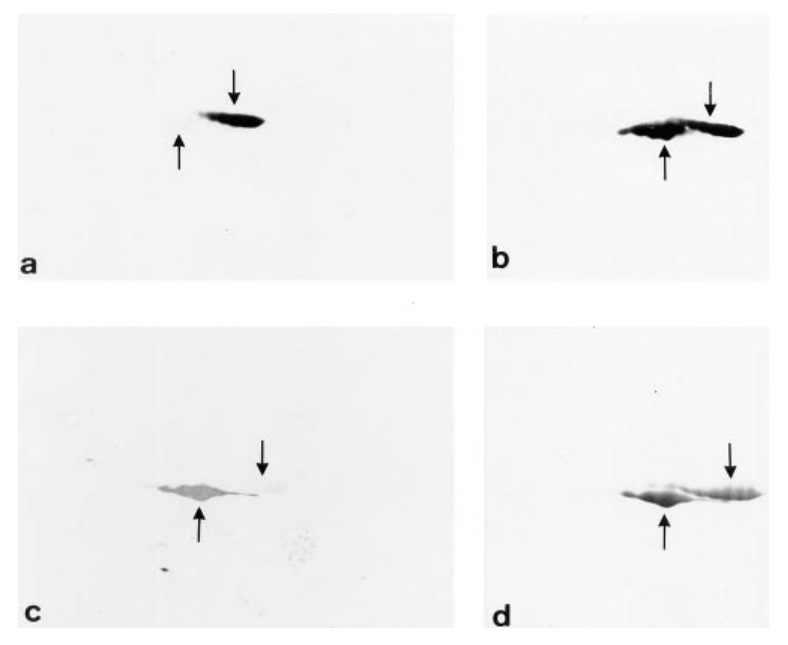

Figure 5. Alkaline phosphatase-coupled goat anti-human IgG (Sigma Chemical Co.) binds to CNPII of the WF $(a)$ when incubated at low dilution (1:750); reincubation with $\mathrm{mAb}$ to $\mathrm{CNP}$ indicates that both CNPII and CNPI are present on the blot $(b)$. (c) NEpHGE blot of the WF (30 $\mu \mathrm{g}$ protein) was incubated with MS serum (diluted 1:5000); the immune reaction with CNPI was detected with alkaline phosphatase-coupled anti-IgM $(1: 10,000)$. (d) Reincubation with $\mathrm{mAb}$ to CNP indicates that both CNPII and CNPI are present on the blot in approximately equal amounts.

C3 fragments, as major proteins copurifying with Ig even when isolations were done under conditions of high stringency, e.g., immune precipitation buffers containing $0.1 \%$ SDS, $1 \%$ Triton $\mathrm{X}-100$, and $1 \%$ deoxycholate (4). Likewise, treatment of purified $\mathrm{IgG}$ with $1 \mathrm{M}$ acetic acid or high concentrations of protein denaturants results in the recovery of $\mathrm{C} 3$ or its various fragments (35). Therefore, we examined which proteins were actually bound to CNPII or CNPI on the nitrocellulose membranes after incubation with normal and MS sera using techniques that were used previously to characterize the binding of $\mathrm{C} 3$ complement to bacterial proteins (36). NEpHGE-resolved CNPI or CNPII were excised from blots, and membranes were blocked to prevent nonspecific binding by added protein (27) and incubated separately with sera from normal donors or patients with MS. Proteins that remained tightly bound to nitrocellulose-immobilized CNP were eluted with $2 \mathrm{M}$ acetic acid, SDS with DTT, or $1 \mathrm{M}$ hydroxylamine at $\mathrm{pH} 9.5$ or 11.5 , separately or sequentially and with the order of elution varied. These elution protocols were chosen to disrupt interactions that were noncovalent in nature, by disulfide bonds or ester linkages, respectively. These are the three well-characterized mechanisms of binding of $\mathrm{C} 3$ to various molecules identified to date (see reference 37). Proteins recovered in the eluates were resolved by SDS-PAGE, stained for protein (Fig. 6, $a$ and $d$ ) or blotted, and probed with antibodies to identify specific proteins (Fig. 6, $b, c, e$, and $f$ ).

Representative results of these analyses are shown in Fig. 6. In Fig. $6 a$, MS serum proteins bound to immobilized CNPII or CNPI were eluted with acetic acid and resolved by SDSPAGE. Recovered proteins were detected by silver staining. The release of bound IgM (indicated as $\mu$ ) exclusively from the CNPI isoform is shown (Fig. $6 a$ ). In Fig. $6 b$, MS serum pretreated with $4 \mathrm{mM}$ DTT was incubated with immobilized 

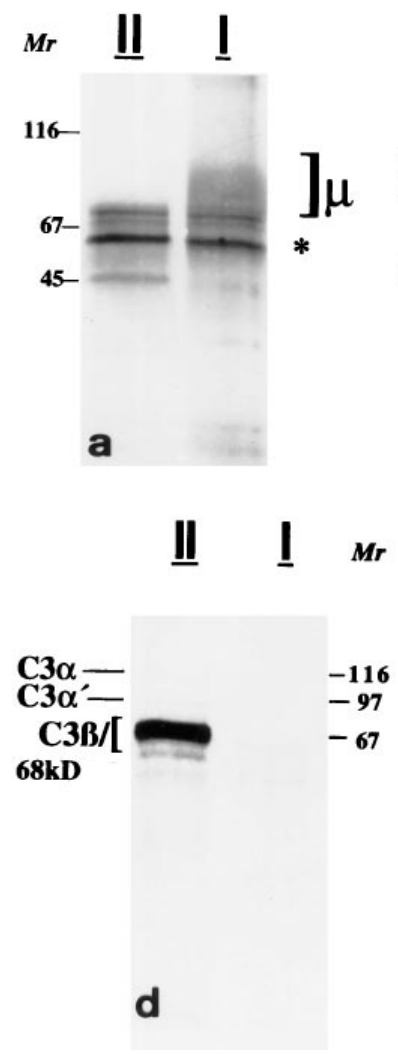
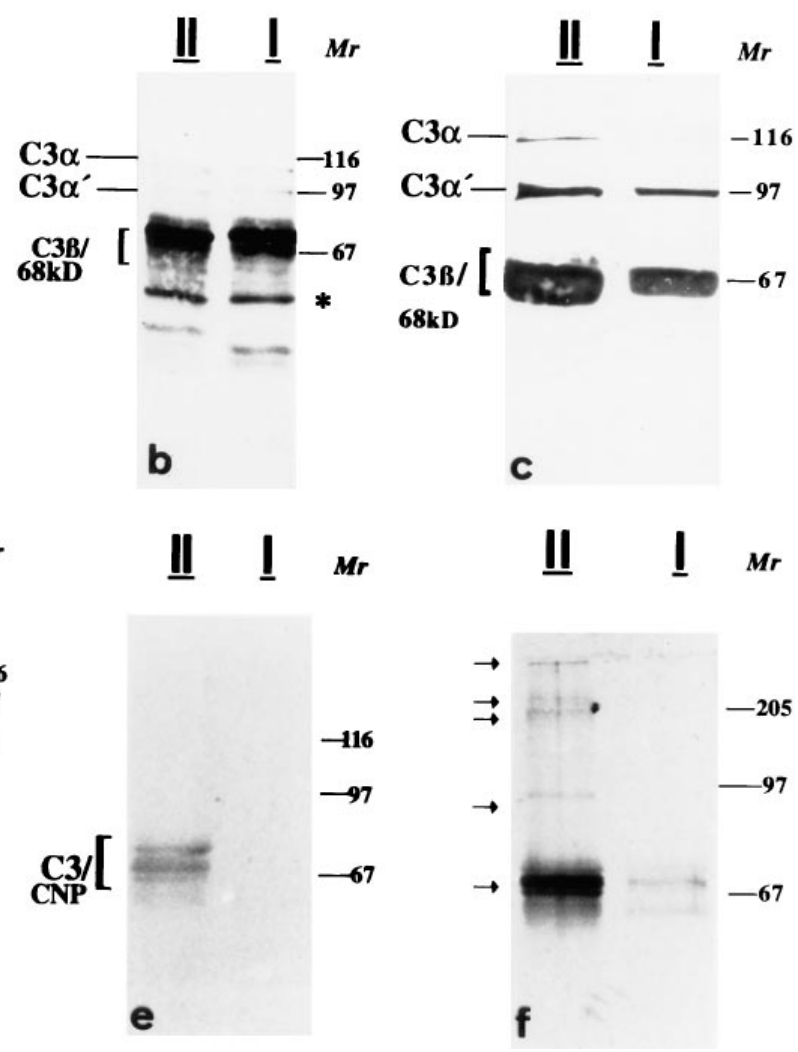

Figure 6. Characterization of the binding of MS Ig or C3 complement from MS and normal serum to CNPI/II. The headings II and $I$ in $a-f$ indicate the eluates were from either CNPII or CNPI. (a) MS serum was incubated with CNPII or CNPI immobilized on nitrocellulose. Bound proteins were eluted with acetic acid, resolved by SDS-PAGE, and detected by silver stain. The bracketed region in $a$ is IgM H chain $(\mu)$; the other bands include eluted CNPII or CNPI, and C3 fragments. The band (*) is also detected when serum is incubated with nitrocellulose pieces without bound protein. (b) Excised CNPI/II was incubated with MS serum adjusted to $4 \mathrm{mM}$ DTT. Bound C3 complement fragments eluted with acetic acid were identified with antiC3 antibody; the positions of $\mathrm{C} 3 \beta(\approx 75$ $\mathrm{kD})$ and the $68-\mathrm{kD}$ degradation product of the $\alpha$ chain $(C 3 \beta / 68 k D)$ overlap extensively. (c) Serum incubated with CNPI/II and eluted as in $a$ were reeluted with 10 $\mathrm{mM}$ Tris- $\mathrm{HCl}, \mathrm{pH} 8$, with $0.5 \%$ SDS, 10 mM DTT. Eluted proteins were resolved by SDS-PAGE, and C3 fragments were identified with anti-C3 antibody. (d) Immobilized CNPI/II incubated with serum was eluted as in $a$ and then reeluted with $1 \mathrm{M}$ hydroxylamine, $\mathrm{pH} 11.5$, and resolved by SDS-PAGE, and proteins were detected

by silver staining. The $\mathrm{C} 3 \beta$ and the $68-\mathrm{kD}$ fragment of the $\alpha$ chain $(C 3 \beta / 68 k D)$ are clearly resolved. ( $e$ and $f$ ) CNPI/II were incubated with normal serum; bound proteins were eluted as in $d$, resolved by SDS-PAGE, blotted, and incubated with antibodies to CNP $(e)$ or C3 $(f)$; the region identified by brackets in $e$ overlaps with the region giving the strongest reaction for $\mathrm{C} 3$ in $f$ (lowest arrow). Numbers on left $(a)$ or right $(b-f)$ indicate molecular masses (in $\mathrm{kD}$ ) of marker proteins.

CNPII or CNPI. Bound proteins were eluted with acetic acid, and $\mathrm{C} 3$ complement protein fragments were identified by immunoblot analysis. In Fig. $6 c$, immobilized CNPII or CNPI, first eluted with acetic acid as shown (Fig. $6 \mathrm{a}$ ), were subsequently eluted with $10 \mathrm{mM}$ Tris $\mathrm{HCl}, \mathrm{pH} 8$, containing $0.5 \%$ SDS and $10 \mathrm{mM}$ DTT. C3 and its fragments were detected by immunoblot analysis as in Fig. $6 \mathrm{~b}$. Membrane-immobilized CNP was eluted with acetic acid and subsequently eluted with $1 \mathrm{M}$ hydroxylamine, $\mathrm{pH}$ 11.5. Hydroxylamine treatment resulted in an additional robust release of $\mathrm{C} 3$ from the CNPII isoform (Fig. $6 d$ ). This result suggests that an ester linkage is the most important mechanism for $\mathrm{C} 3$ binding to CNPII. In contrast, the hydroxylamine treatment did not result in the release of any detectable C3 from CNPI. Cumulatively, these results indicate tenacious binding of $\mathrm{C} 3$ to $\mathrm{CNP}$, variously mediated by noncovalent interactions, linkage involving disulfide bonds, or ester linkages. Parallel blots of eluates were also probed with antibodies to CNP (Fig. $6 e$ ) and C3 (Fig. $6 f$ ). Several bands with identical mobility were identified which were reactive with both antisera, consistent with cross-links containing $\mathrm{CNP}$ or $\mathrm{CNP}$ fragments and $\mathrm{C} 3$ which were resistant to cleavage with SDS-DTT or hydroxylamine. Bands ranging from $>200$ to $105 \mathrm{kD}$ were detected most clearly with anti-C3 antibody (Fig. $6 f$ ). Identical results were obtained using MS or non-MS sera with respect to $\mathrm{C} 3$ binding. In contrast, bound IgM was also recovered in eluates of MS sera and exclusively from the CNPI isoform (Fig. $6 a$ ). We also probed eluates for the presence of $\mathrm{C} 1 \mathrm{q}$ but none was detected, consistent with earlier reports that protein moieties of myelin other than CNP mediated the binding of C1q (38). However, a candidate C1qbinding protein, migrating close to but well-resolved from $\mathrm{CN}$ PII using NEpHGE, has been identified, and its characterization is currently underway (data not shown).

CNPI of human peripheral nerve does not bind MS IgM. $\mathrm{CNP}$ is expressed in the PNS, albeit at levels $\sim 10$-fold less than in the CNS (11). As symptomatic involvement of the PNS is exceedingly rare in MS, we wanted to see if the epitope recognized by MS sera on CNS CNPI was present on PNS CNPI. Human PNS myelin was isolated and resolved by NEpHGE (Fig. $7 a$ ), and CNP was detected after electroblotting (Fig. 7 b). Fig. $7 c$ shows that the CNP isoforms of human PNS resolved by SDS-PAGE are present in $\approx 1: 1$ stoichiometry as in human CNS myelin, although by NEpHGE the amount of PNS CNPII that migrates fully into the gel (Fig. $7 a$ ) is less than for CNPI. Blotted PNS myelin was probed using the MS sera that gave the strongest reaction with CNS CNP. No response to PNS CNPI was observed, indicating that the epitope recognized by MS sera was unique to CNS CNPI and not expressed at detectable levels on PNS CNPI.

\section{Discussion}

The IgM response and $M S$. The identification of CNPI as a target of the humoral immune response represents the first 

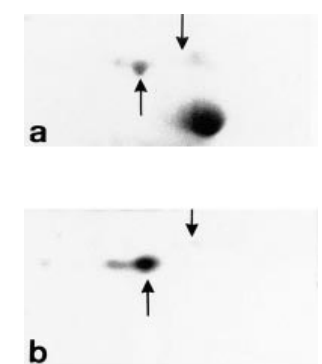

b

Figure 7. NEpHGE separation and immunoblot analysis of proteins of the PNS myelin; (a) portions of gel stained for protein indicate the positions of PNS CNPI $(\uparrow)$ and CNPII $(\downarrow)$, respectively. In $b$, the CNP isoforms were detected using rabbit polyclonal antibody to CNP; significantly less CNPII compared with CNPI enters the NEpHGE gel. (c) SDS-PAGE separation of PNS myelin and immunoblot analysis with $\mathrm{mAb}$ to CNP showing equal intensity of reaction for both CNP isoforms.

molecular characterization of an IgM antigen in MS. Although, appropriately, most emphasis has been directed to analysis of the IgG response in MS since first identified (39), there is considerable evidence for IgM disturbances in MS. IgM is a putative marker for an active or persistent viral infection in MS and may also be a probe for nonpeptide antigens which are now implicated in a diverse and increasing number of autoimmune and postinfectious disorders of peripheral nerve, many of which are demyelinating in nature (34). IgM lymphocytotoxic antibodies were found in $67 \%$ of MS patients compared with $8 \%$ of controls; it was hypothesized that such antibodies might be a marker for viral infection in MS (40). CNP is highly expressed in lymphocytes (13); thus, the lymphocytotoxic antibodies may be related to the IgM anti-CNPI response reported here. CSF IgM is increased in $\approx 65 \%$ of patients with MS, with the mean CSF IgM actually $\approx 10$-fold elevated compared with normal patients (30). Despite this, CSF IgM levels are not quantitatively striking compared with CSF IgG levels. However, pentameric (or hexameric) $\operatorname{IgM}$ has an apparent $M_{\mathrm{r}} \approx 10^{6}$, and its egress from the brain interstitium into CSF and the CNS extracellular spaces is pore restricted. Thus, CSF levels are marginally informative and may mislead about the total IgM burden in MS CNS. This is in contrast to IgG, where a linear correlation of MS CSF and CNS IgG levels has been observed (41). Nevertheless, oligoclonal IgM bands were detected in the CSF from 55\% of MS patients (42). We reported that a considerable portion of the MS CNS Ig which is not aqueous soluble and sediments with the membrane fraction, requiring detergent for solubilization, is $\operatorname{IgM}(4)$. Subsequently, we found that a considerable amount of IgM in MS CNS is also resistant to solubilization in buffer containing $4 \%$ NP-40, which quantitatively solubilizes many transmembrane glycoproteins, including HLA-DR in MS CNS (our unpublished results), with the possible implication that this IgM fraction may also be immune complexed with specific antigen(s).

IgM, CNP, and MS etiopathogenesis. Immune electron microscopy demonstrates that CNP is apposed to the cytoplasmic leaflet of the OL membrane. In the mature OL/myelin membrane, CNP is located in OL processes and the paranodal loops of the myelin sheath, but not in compact myelin (43). This localization does not readily permit interactions in vivo between CNP and the specific anti-CNPI antibodies reported here. Nevertheless, there are several ways that IgM antibodies might be accessible to CNP. First, the OL/myelin membrane has one or several molecules that activate the classical comple- ment pathway (18). The formation of the complement membrane attack complex in MS CNS, for which there is biochemical and immunocytochemical evidence (44), might permit intracellular penetration of Ig. Likewise, T cell perforins might also compromise OL membrane integrity with the same result. Second, the multilamellar myelin sheath including all its protein components is metabolically dynamic. Protein turnover in myelin is $\approx 40 \%$ that of whole brain, with CNPII/I most active in terms of incorporation and turnover of labeled amino acid (45). The resident microglia may regulate this turnover, a situation where $\mathrm{C} 3$ complement may become accessible to $\mathrm{CNP}$ facilitating myelin phagocytosis by microglia as an aspect of glial cell regulation of the myelination status of normal brain. In addition, there is measurable CNPase activity in CSF and serum in normal patients as well as patients with various neurological diseases which may originate from the OL/myelin membrane or lymphocytes $(11,46)$. Prineas and colleagues examined fresh lesions in the brain and spinal cord of patients with MS who died within several weeks after the onset of the first symptoms. Myelin debris localized within macrophages stained positive for CNP, including macrophages that had entered the Virchow-Robin spaces. Cells with OL-like morphology were observed in large numbers throughout fresh lesions after acute myelin breakdown and before new myelin formation. These were highly immunoreactive for CNP, but not myelin-associated glycoprotein or MBP, suggesting they were OL precursors that when terminally differentiated, mediated the remyelination that occurs in the early phases of MS (47). AntiCNPI antibodies identified in our study, as well as potent binding of $\mathrm{C} 3$ to $\mathrm{CNP}$ in this setting, may be a pivotal component of the opsonic phagocytosis of myelin that appears to be the fundamental molecular mechanism by which demyelination proceeds in MS $(48,49)$. Separate from any role in the mediation of demyelination, the antibody response to CNPI may represent molecular mimicry. As with many viral proteins, CNP is extensively and differentially posttranslationally modified. It is possible that these modifications are shared with proteins of virus(es) that may be involved in MS. The viruses of chronic hepatitis are associated with production of $\operatorname{IgM}$ antibodies, and assays for antiviral IgM are useful to monitor disease. In chronic infection with hepatitis $\mathrm{C}$ virus, persistence of specific IgM antibody is indicative of active viral replication, and decreases in virus-specific IgM correlates with response to IFN- $\alpha$ (50). These observations may have relevance to MS in two specific contexts, i.e., the use of interferons as MS therapies and $\mathrm{MS}$ as a disease in which a virus may be involved.

CNP expression and retinal periphlebitis in MS. One particularly perplexing aspect of MS has been the common occurrence of a periphlebitis reaction in the retina (51), a location where myelin is absent. CNP is expressed in retina principally in photoreceptors at about the same level as in CNS white matter (14). Cell death occurs in the retina during development and continues postmitotically. The resident microglia are the major phagocytic cells for dying retinal cells (52). Human retinal microglia are phenotypically similar to dendritic antigen-presenting cells and constitutively express class I/II molecules (53). Based on these observations, we propose that the immune response to CNPI identified in this study is related to the retinal manifestations that are a common feature of MS. CNP is present also in thymus and lymphocytes (13), which may account for the presence of anti-CNPI antibody in sera from MS patients. This widespread expression of class I/II as 
well as nonclassical MHC molecules such as CD1, in conjunction with enhanced antigen presentation due to specific antibody (54), represents a mechanism whereby CNP-derived (lipo-) peptides may be presented for immune surveillance not only to CD4+ or CD8+ T cells but possibly also to $\mathrm{T} \gamma / \delta$ cells which are prominent in MS lesions (55) and preferentially recognize nonpeptide antigens (56) including the types of moieties by which CNP is modified posttranslationally. The expression of CNP by lymphocytes may likewise have relevance to the cellular immune derangements described in peripheral blood of MS patients $(20,21)$.

Antibodies to CNPI and clinical applications. Here, we have reported on the immune response to CNPI only. The methodological approaches presented here offer promise that analysis of the humoral immune response in MS for antigenic specificity can now proceed expeditiously, leading to identification of major targets for the humoral response in MS. Furthermore, if opsonic phagocytosis by macrophages is the fundamental determinant of the demyelination that occurs in MS $(48,49)$, the observation that CNP binds C3 complement tenaciously might provide an option to explore therapeutic interventions which block this interaction. In this context, treatment with soluble human complement receptor 1 inhibited CNS inflammation and almost completely blocked demyelination in a model of demyelination with similarities to MS (57). During the course of our studies, other specificities for MS Ig as well as Ig from normal subjects were discovered. Undoubtedly, these represent part of the natural autoantibody repertoire but may nevertheless be relevant to MS symptomatology and pathology in the context of BBB disturbance, permitting the accumulation of specific Ig in the brain parenchyma $(58,59)$. Only the immune response to CNPI has been identified so far as having such high specificity for MS. From a clinical standpoint, it is now important to achieve a molecular characterization of the epitope of CNPI responsible for the IgM immunoreactivity as an approach to developing a sensitive and quantitative immunoassay. From a research standpoint, the antibody response might profitably be examined as a probe for an infectious agent in MS.

\section{Acknowledgments}

We are grateful to Drs. S. Morgello, J. Moreno, P. Werner, and H. Kaufman for careful reading of the manuscript and valuable suggestions, and the staff of the National Neurological Research Bank (Los Angeles, CA) for their assistance in providing biological materials used in the study.

Supported in part by National Institutes of Health grant AI-42088 (to M.J. Walsh).

\section{References}

1. Martin, R., H.F. McFarland, and D.E. McFarlin. 1992. Immunological aspects of demyelinating diseases. Annu. Rev. Immunol. 10:153-187.

2. Bansil, S., S.D. Cook, and C. Rohowsky-Kochan. 1995. Multiple sclerosis: immune mechanisms and update on current therapies. Ann. Neurol. 37:S87S101.

3. Walsh, M.J., W.W. Tourtellotte, J. Roman, and W. Dreyer. 1985. Immunoglobulin $\mathrm{G}, \mathrm{A}$, and $\mathrm{M}$-clonal restriction in multiple sclerosis cerebrospinal fluid and serum-analysis by two-dimensional electrophoresis. Clin. Immunol. Immunopathol. 35:313-327.

4. Walsh, M.J., and W.W. Tourtellotte. 1986. Temporal invariance and clonal uniformity of brain and cerebrospinal $\operatorname{IgG}, \operatorname{IgA}$, and $\operatorname{IgM}$ in multiple sclerosis. J. Exp. Med. 163:41-53.

5. Colombo, E., K. Banki, A.H. Tatum, J. Daucher, P. Ferrante, R.S. Mur- ray, P.E. Phillips, and A. Perl. 1997. Comparative analysis of antibody and cellmediated autoimmunity to transaldolase and myelin basic protein in patients with multiple sclerosis. J. Clin. Invest. 99:1238-1250.

6. Miller, D.H., P.S. Albert, F. Barkhof, G. Francis, J.A. Frank, S. Hodgkinson, F.D. Lublin, D.W. Paty, S.C. Reingold, and J. Simon. 1996. Guidelines for the use of magnetic resonance techniques in monitoring the treatment of multiple sclerosis. Ann. Neurol. 39:6-16.

7. Baekkeskov, S., H.J. Aanstoot, S. Christgau, A. Reetz, M. Solimena, M. Cascalho, F. Folli, H. Richter-Oleson, and P. DeCamilli. 1990. Identification of the $64 \mathrm{kD}$ autoantigen in insulin-dependant diabetes as the GABA-synthesizing enzyme glutamic acid decarboxylase. Nature. 347:151-157.

8. Pozzilli, P. 1996. Prevention of insulin-dependant diabetes: where are we now? Diabetes Metab. Rev. 12:127-135.

9. Challoner, P.B., K.T. Smith, J.D. Parker, D.L. MacLeod, S.N. Coulter, T.M. Rose, E.R. Schultz, J.L. Bennett, R.L. Garber, M. Chang, et al. 1995. Plaque-associated expression of human herpesvirus 6 in multiple sclerosis. Proc. Natl. Acad. Sci. USA. 92:7440-7444.

10. Sanders, V.J., A.E. Waddell, S.L. Felisan, X. Li, A.J. Conrad, and W.W. Tourtellotte. 1996. Herpes simplex virus in postmortem multiple sclerosis brain tissue. Arch. Neurol. 53:125-133.

11. Sprinkle, T.J. 1989. 2', 3'-cyclic nucleotide $3^{\prime}$-phosphodiesterase, an oligodendrocyte-Schwann cell and myelin-associated enzyme of the nervous system. Crit. Rev. Neurobiol. 4:235-301.

12. Thompson, R.J. 1992. 2',3'-cyclic nucleotide-3'-phosphohydrolase and signal transduction in central nervous system myelin. Biochem. Soc. Trans. 20: $621-626$.

13. Bernier, L., F. Alvarez, M. Norgard, D.W. Raible, A. Mentaberry, J.G Schembri, D.D. Sabatini, and D.R. Colman. 1987. Molecular cloning of a 2',3'cyclic nucleotide $3^{\prime}$-phosphodiesterase: mRNAs with different $5^{\prime}$ ends encode the same set of proteins in nervous and lymphoid tissues. J. Neurosci. 7:27032710 .

14. Giulian, D., and S. Moore. 1980. Identification of $2^{\prime}, 3^{\prime}$ cyclic nucleotide $3^{\prime}$-phosphodiesterase in the vertebrate retina. J. Biol. Chem. 255:5993-5995.

15. Kurihara, T., Y. Tohyama, J. Yamamoto, T. Kanamatsu, R. Watanabe, and S. Kitajima. 1992. Origin of brain 2',3'-cyclic-nucleotide $3^{\prime}$-phosphodiesterase doublet. Neurosci. Lett. 138:49-52.

16. Agrawal, H.C., T.J. Sprinkle, and D. Agrawal. 1990. 2',3'-cyclic nucleotide $3^{\prime}$-phosphodiesterase in the central nervous system is fatty-acylated by thioester linkage. J. Biol. Chem. 265:11849-11853.

17. Cox, M.E., E.N. Gao, and P.E. Braun. 1994. C-terminal CTII motif of $2^{\prime}, 3^{\prime}$-cyclic nucleotide $3^{\prime}$-phosphodiesterase undergoes carboxylmethylation. $J$. Neurosci. Res. 39:513-518.

18. Shin, M.L., and C.L. Koski. 1992. The complement system in demyelination. In Myelin: Biology and Chemistry. R.E. Martenson, editor. CRC Press, Inc., Boca Raton, FL. 801-831.

19. Piddlesden, S.J., H. Lassmann, F. Zimprich, B.P. Morgan, and C. Linington. 1993. The demyelinating potential of antibodies to myelin oligodendrocyte glycoprotein is related to their ability to fix complement. Am. J. Pathol. 143:555-564.

20. Hafler, D.A., D.A. Fox, M.E. Manning, S.F. Schlossman, E.L. Reinherz, and H.L. Weiner. 1985. In vivo activated T lymphocytes in the peripheral blood and cerebrospinal fluid of patients with multiple sclerosis. N. Engl. J. Med. 312: 1405-1411.

21. Bongioanni, P., C. Fioretti, R. Vanacore, F. Bianchi, F. Lombardo, F. Ambrogi, and G. Meucci. 1996. Lymphocyte subsets in multiple sclerosis: a study with two-color fluorescence analysis. J. Neurol. Sci. 139:71-77.

22. O'Farrell, P.Z., H.M. Goodman, and P.H. O'Farrell. 1977. High resolution two-dimensional electrophoresis of basic as well as acidic proteins. Cell. 12: 1133-1142.

23. Walsh, M.J., J. McDougall, and B. Wittmann-Liebold. 1988. Extended $\mathrm{N}$-terminal sequencing of proteins of archaebacterial ribosomes blotted from two-dimensional gels onto glass fiber and poly(vinylidene difluoride) membrane. Biochemistry. 27:6867-6876.

24. Norton, W.T. 1974. Isolation of myelin from nerve tissue. Methods Enzymol. 31:435-444.

25. Walsh, M.J., and N. Kuruc. 1992. The postsynaptic density: constituent and associated proteins characterized by electrophoresis, immunoblotting, and peptide sequencing. J. Neurochem. 59:667-678.

26. Gorga, J.C., V. Horejsi, D.R. Johnson, R. Raghupathy, and J.L. Strominger. 1987. Purification and characterization of class II histocompatibility antigens from a homozygous human B cell line. J. Biol. Chem. 262:1608716094.

27. Aebersold, R.H., J. Laevitt, R.A. Saavedra, L.E. Hood, and S.B.H. Kent. 1987. Internal amino acid sequence analysis of proteins separated by onedimensional or two-dimensional electrophoresis after in situ protease digestion on nitrocellulose. Proc. Natl. Acad. Sci. USA. 84:6970-6974.

28. Sadegh-Nasseri, S., L.J. Stern, D.C. Wiley, and R.N. Germain. 1994. MHC class II function preserved by low-affinity peptide interactions preceding stable binding. Nature. 370:647-650.

29. De Angelis, D.A., and P.E. Braun. 1996. 2',3'-Cyclic nucleotide $3^{\prime}$-phosphodiesterase binds to actin-based cytoskeletal elements in an isoprenylationindependent manner. J. Neurochem. 67:943-951. 
30. Forsberg, P., A. Henriksson, H. Link, and S. Ohman. 1984. Reference values for CSF-IgM, CSF-IgM/S-IgM ratio and IgM index, and its application to patients with multiple sclerosis and aseptic meningoencephalitis. Scand. J. Clin. Lab. Invest. 44:7-12.

31. Eisenberg, R. 1976. The specificity and polyvalency of binding of a monoclonal rheumatoid factor. Immunochemistry. 13:355-359.

32. Roubey, R.A.S. 1994. Autoantibodies to phospholipid-binding plasma proteins: a new view of lupus anticoagulants and other "antiphospholipid" autoantibodies. Blood. 84:2854-2867.

33. Nores, G.A., T. Dohi, M. Taniguchi, and S. Hakomori. 1987. Densitydependent recognition of cell surface GM3 by a certain anti-melanoma antibody, and GM3 lactone as a possible immunogen: requirements for tumor-associated antigen and immunogen. J. Immunol. 139:3171-3176.

34. Latov, N. 1995. Pathogenesis and therapy of neuropathies associated with monoclonal gammopathies. Ann. Neurol. 37:S32-S42.

35. Nezlin, R., A. Freywald, and M. Oppermann. 1993. Proteins separated from human IgG molecules. Mol. Immunol. 30:935-940.

36. Newman, S.L., and L.K. Mikus. 1985. Deposition of C3b and iC3b onto particulate activators of the human complement system. Quantitation with monoclonal antibodies to human C3. J. Exp. Med. 161:1414-1431.

37. Lambris, J.D. 1988. The multifunctional role of C3, the third component of complement. Immunol. Today. 9:387-393.

38. Vanguri, P., and M.L. Shin. 1986. Activation of complement by myelin: identification of C1-binding proteins of human myelin from central nervous tissue. J. Neurochem. 46:1535-1541.

39. Kabat, E.A., D.A. Freedman, J.P. Murray, and V. Knaub. 1950. A study of the crystalline albumin, gamma globulin and total protein in the cerebrospinal fluid of one hundred cases of multiple sclerosis and in other diseases. Am. J. Med. Sci. 219:55-64.

40. Schocket, A.L., and H.L. Weiner. 1978. Lymphocytotoxic antibodies in family members of patients with multiple sclerosis. Lancet. 1:571-573.

41. Tourtellotte, W.W., and J.A. Parker. 1966. Multiple sclerosis: correlation between immunoglobulin G in cerebrospinal fluid and brain. Science. 154: 1044-1046.

42. Sharief, M.K., and E.J. Thompson. 1991. Intrathecal immunoglobulin M synthesis in multiple sclerosis. Relationship with clinical and cerebrospinal fluid parameters. Brain. 114:181-195.

43. Trapp, B.D., L. Bernier, S.B. Andrews, and D.R. Colman. 1988. Cellular and subcellular distribution of $2^{\prime}, 3^{\prime}$-cyclic nucleotide $3^{\prime}$-phosphodiesterase and its mRNA in the rat central nervous system. J. Neurochem. 51:859-868.

44. Compston, D.A.S., B.P. Morgan, A.K. Campbell, P. Wilkins, G. Cole, N.D. Thomas, and B. Jasini. 1989. Immunocytochemical localization of the terminal complement complex in multiple sclerosis. Neuropathol. Appl. Neurobiol. 15:307-316.

45. Lajtha, A., J. Toth, K. Fujimoto, and H.C. Agrawal. 1977. Turnover of myelin proteins in mouse brain in vivo. Biochem. $J$. 164:323-329.
46. Clapshaw, P.A., H.W. Muller, H. Weitholter, and W. Seifert. 1984. Simultaneous measurement of $2^{\prime}, 3^{\prime}$ cyclic-nucleotide $3^{\prime}$ phosphodiesterase and RNase activities in sera and spinal fluids of multiple sclerosis patients. J. Neurochem. 42:12-15.

47. Prineas, J., E. Kwon, P. Goldenberg, A. Ilyas, R. Quarles, J. Benjamins, and T. Sprinkle. 1989. Multiple sclerosis: oligodendrocyte proliferation and differentiation in fresh lesions. Lab. Invest. 61: 489-503.

48. Prineas, J., and B. Graham. 1980. Multiple sclerosis: capping of surface immunoglobulin $\mathrm{G}$ on macrophage engaged in myelin breakdown. Ann. Neurol. 2:149-158.

49. Sriram, S., and M. Rodriguez. 1997. Indictment of the microglia as the villain in multiple sclerosis. Neurology. 48:464-470.

50. Yuki, N., N. Hayashi, K. Ohkawa, H. Hagiwara, M. Oshita, K. Katayama, Y. Sasaki, A. Kasahara, H. Fusamoto, and T. Kamada. 1995. The significance of immunoglobulin $\mathrm{M}$ antibody response to hepatitis $\mathrm{C}$ virus core protein in patients with chronic hepatitis C. Hepatology. 22:402-406.

51. Tola, M.R., E. Granieri, I. Casetta, P. Monari, R. Scorrano, V. Mazzeo, E. Paolino, V.C. Monetti, and V. Govoni. 1993. Retinal periphlebitis in multiple sclerosis: a marker of disease activity. Eur. Neurol. 33:93-96.

52. Egensperger, R., J. Maslim, S. Bisti, H. Holländer, and J. Stone. 1996. Fate of DNA from retinal cells dying during development: uptake by microglia and macroglia (Müller cells). Dev. Brain Res. 97:1-8.

53. Penfold, P.L., J.M. Provis, and S.C. Liew. 1993. Human retinal microglia express phenotypic characteristics in common with dendritic antigen-presenting cells. J. Neuroimmunol. 45:183-191.

54. Simitsek, P.D., D.G. Campbell, A. Lanzavecchia, N. Fairweather, and C. Watts. 1995. Modulation of antigen processing by bound antibodies can boost or suppress class II major histocompatibility complex presentation of different T cell determinants. J. Exp. Med. 181:1957-1963.

55. Selmaj, K., C.F. Brosnan, and C.S. Raine. 1991. Colocalization of lymphocytes bearing $\gamma / \delta \mathrm{T}$-cell receptor and heat shock protein hsp65+ oligodendrocytes in multiple sclerosis. Proc. Natl. Acad. Sci. USA. 88:6452-6456.

56. Tanaka, Y., C.T. Morita, Y. Tanaka, E. Nieves, M.B. Brenner, and B.R. Bloom. 1995. Natural and synthetic non-peptide antigens recognized by human gamma delta T cells. Nature. 375:155-158.

57. Piddlesden, S.J., M.K. Storch, M. Hibbs, A.M. Freeman, H. Lassmann, and B.P. Morgan. 1994. Soluble recombinant complement receptor 1 inhibits inflammation and demyelination in antibody-mediated demyelinating experimental allergic encephalomyelitis. J. Immunol. 152:5477-5484.

58. Kermode, A.G., A.J. Thompson, P. Tofts, D.G. MacManus, B.E. Kendall, D.P. Kingsley, I.F. Moseley, P. Rudge, and W.I. McDonald. 1990. Breakdown of the blood-brain barrier precedes symptoms and other MRI signs of new lesions in multiple sclerosis. Pathogenetic and clinical implications. Brain. 113:1477-1489.

59. Gay, D., and M. Esiri. 1991. Blood-brain barrier damage in acute multiple sclerosis plaques: an immunocytologic study. Brain. 114:557-572. 